\title{
The Nature and Treatment of Pandemic-Related Psychological Distress
}

\author{
William C. Sanderson ${ }^{1}$ (D) Vinushini Arunagiri ${ }^{1} \cdot$ Allison P. Funk $^{1} \cdot$ Karen L. Ginsburg ${ }^{1} \cdot$ Jacqueline K. Krychiw $^{1}$. \\ Anne R. Limowski ${ }^{1}$. Olenka S. Olesnycky ${ }^{1} \cdot$ Zoe Stout $^{1}$
}

Published online: 27 June 2020

(c) Springer Science+Business Media, LLC, part of Springer Nature 2020

\begin{abstract}
The COVID-19 crisis has created a "mental health pandemic" throughout the world. Scientific data are not available to fully understand the nature of the resulting mental health impact given the very recent onset of the pandemic, nevertheless, there is a need to act immediately to develop psychotherapeutic strategies that may alleviate pandemic-related distress. The psychological distress, in particular fear and sadness, is a function of the pandemic's negative impact upon people's ability to meet their most basic needs (e.g., physical safety, financial security, social connection, participation in meaningful activities). This paper presents evidence-based cognitive behavioral strategies that should prove useful in reducing the emotional suffering associated with the COVID crisis.
\end{abstract}

Keywords COVID-related psychological distress $\cdot$ Pandemic $\cdot$ Psychological intervention

Mental health issues such as anxiety, depression, substance abuse, and suicide were increasing in the U.S. well before the pandemic (Substance Abuse and Mental Health Services Administration 2019). Thus, it is no surprise that the very recent "mental health pandemic" as a result of COVID-19 (COVID) will further accelerate the increasing prevalence of these disorders (Marques et al. 2020; Strakowski et al. 2020) as a result of its impact upon people's day-to-day functioning (Brooks et al. 2020). Indeed, a recent survey by the Kaiser Family Foundation found that 56 percent of people reported that the worry or stress tied to COVID had a negative effect on their emotional well-being (Panchal et al. 2020). Moreover, many individuals may attempt to cope with these negative psychological effects in unhelpful ways (e.g., alcohol/ substance use) that can actually result in an increased susceptibility to COVID-19 (Da et al. 2020; Volkow 2020). Clearly, the COVID crisis is likely to have an enormous negative impact upon mental health if left ignored.

The pandemic has disrupted the ability to satisfy core human needs in almost all areas to an alarming extent (e.g.,

Co-authors (all authors other than the primary author) listed in alphabetical order to demonstrate equal contribution.

William C. Sanderson

william.c.sanderson@hofstra.edu

1 Department of Psychology, Hofstra University, Hempstead, NY 11549, USA
Brooks et al. 2020). Much of the population is worried about meeting their most basic needs (e.g., paying rent, buying food) as a result of economic hardship (e.g., unemployment) as well as an increasing scarcity of resources (e.g., food, cleaning products, personal protective equipment such as masks). People are living in a chronic state of fear of contracting the virus. Socially distancing and disconnecting rather than affiliating has increased loneliness and decreased social support, particularly in elderly and disabled populations and those with underlying health conditions. The artificial substitutes for social connection such as online video events often exacerbate, rather than relieve, the feeling of disconnection. People have been removed from the sources of activity that allow them to feel esteem, status, and pleasure (e.g., work, graduation ceremonies, weddings, engaging in sporting activities). The impact upon dating and finding a mate is profound in an era of remaining six feet from others and fearing any close contact may lead to transmission of the virus. Finally, for those who are parents, the stress of this role is enormous (e.g., home schooling, explaining the pandemic to young children), and certainly the idea that children will flourish in the present environment seems implausible.

Given the sudden onset and impact of the pandemic, scientific data are not available to fully understand the nature of the resulting psychological distress, but existing research suggests anxiety, depression, and worsening stress are common reactions (Rajkumar 2020). People are suffering and 
reaching out for assistance- - there was a need to act immediately in order to develop strategies that may alleviate psychological distress. Typically, when we produce a paper or treatment manual we spend months planning, writing, and reviewing it before making it available to others. Given the rapidly evolving mental health crisis that occurred, we produced an initial product after only 3 weeks of the first coronavirus cases in New York, which we continued to revise as we learned more about Covid-related problems (cf. Sanderson et al. 2020).

To develop this guide, we brainstormed using our clinical observation skills to identify pandemic-related triggers and resultant psychological problems we were seeing in our patients, family, friends-and our own lives. Thus, we are confident that the areas identified in the original document (Sanderson et al. 2020) represent the spectrum of COVID related psychological distress. Once we created this list we developed strategies that would allow people to manage these negative emotional states using a self-help format (this guide can be accessed at www.psychrescue-covid 19.com or at the permanent DOI address provided in the reference section). This self-help guide can be used in conjunction with the current paper, which more concisely outlines therapeutic strategies, to maximize the effectiveness of one's intervention. Nearly all of the strategies that we included were taken from evidence-based psychological treatments for individuals with anxiety disorders, depression, and related emotional distress (Table 1). The approach we used is identical to that which led to the development of a variety of evidence-based treatment manuals for psychological disorders (e.g., panic disorder, depression) where basic cognitive behavioral strategies were tailored to address the specific psychopathology of the disorder. We expected that these strategies would prove useful for those suffering from similar emotional states secondary to the challenges associated with the COVID crisis. In fact, since so many individuals are struggling as a direct result of the COVID crisis, we have started to conceptualize these symptom clusters as a Pandemic-Related Stress Disorder (PRSD).

While there has been progress, as outlined above, in understanding the nature and interventions for COVID related psychological distress, an additional problem has emerged as a result: the already overburdened mental health system must now provide treatment for a substantial wave of persons in need. Similar to the concern of flattening the curve for medical disorders to avoid overburdening the healthcare system, mental healthcare may find itself in the position of having more patients than can be reasonably accommodated (Marques et al. 2020). In fact, it is probable that this substantial increase in patients will last well beyond the pandemic itself (Fiorillo and Gorwood 2020). Many individuals, particularly younger generations, are

Table 1 Summary of commonly encountered distress provoking triggers and recommended evidence based therapeutic strategies

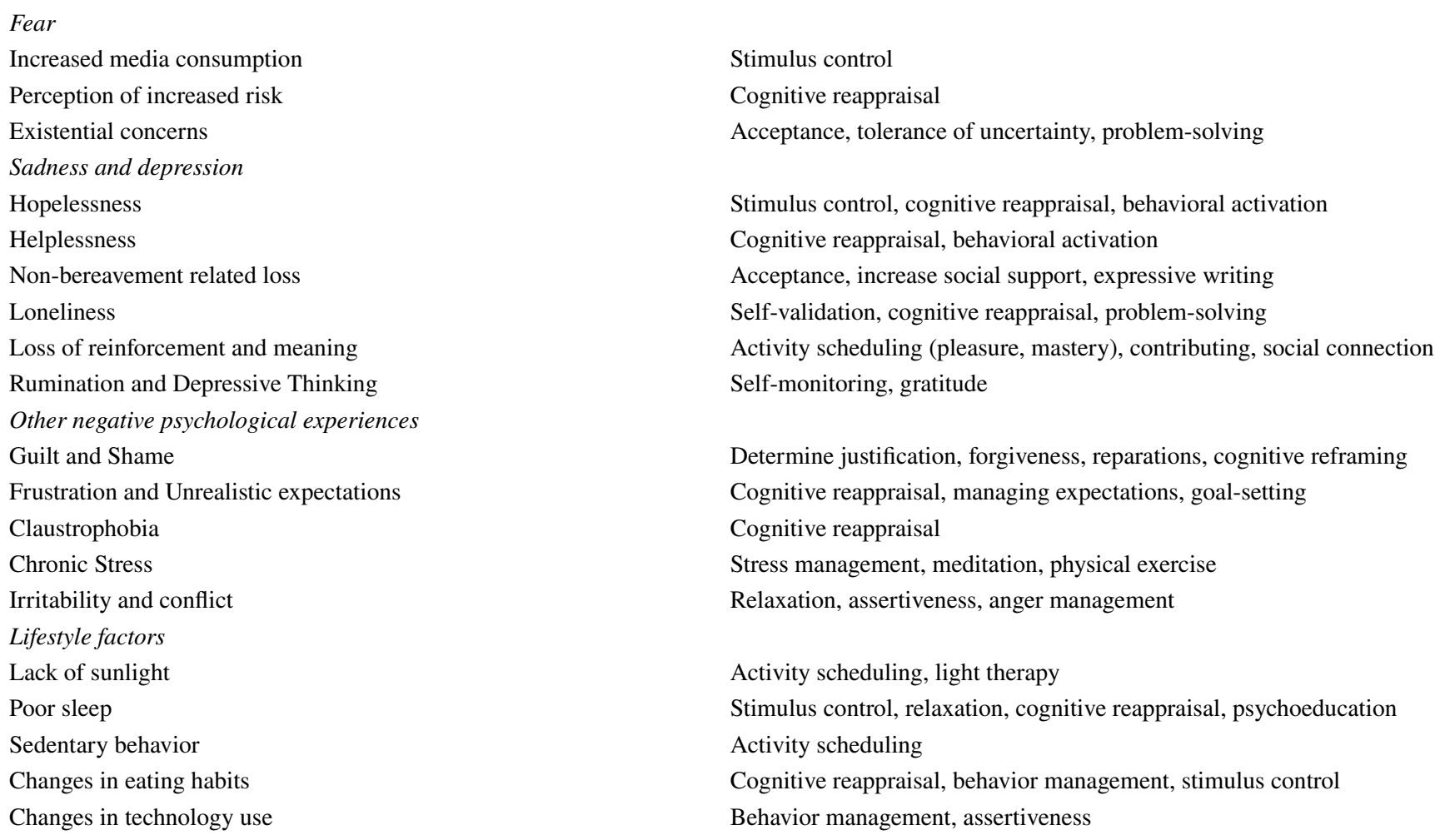


experiencing significant consequences of the economic crisis (e.g., financial uncertainty) that will take considerable time to resolve. A national survey conducted by The National Center for Health Statistics indicated that approximately $35 \%$ of Americans reported experiencing symptoms of an anxiety or depressive disorder during the end of May into June 2020, when several states had already started to reopen (CDC 2020). Furthermore, the process of reopening in itself may result in increased feelings of anxiety. As individuals have become accustomed to staying at home for safety, beginning to reintegrate into society is nerve-wracking for many; psychologists have started to refer to these fears as "re-entry anxiety" (Dusharme 2020). Moreover, the Centers for Disease Control and Prevention alerted that the second wave of COVID-19 is likely to be far more devastating than the first (Sun 2020).

As a result, in line with the suggestions of Marques et al. (2020), we have been examining scalable psychotherapeutic intervention models to increase our proactivity, reach out to those with distress, and attempt to decrease the likelihood of more severe responses later on. Schleider (2020) has developed and tested brief, accessible interventions delivered in non-traditional settings (i.e., telepsychology). She found that a single session of a solution-focused consultation was associated with a reduction in psychological distress in adults seeking psychotherapy (Schleider et al. 2020). Thus, we adopted her approach and have developed and are in the process of field-testing a consultation-oriented intervention (rather than a more traditional "psychotherapy" model) consisting of two 60-min telepsychology online video sessions (cf. Limowski et al. 2020). The focus of the first session is to identify factors that appear to be causing the most distress and providing evidence-based strategies for the client to utilize. The focus of the second session, held approximately one week later, is to assess the success of the initial recommendations and to provide additional strategies if necessary. Based upon the results of our field-testing we may ultimately increase the number of sessions in this intervention.

\section{Fear}

Fear is an emotion provoked by the perception of danger or threat that evolved to protect us and enhance our ability to survive. Given that the core concerns of this pandemic are about illness and death, fear is an expected emotion. The first step in working with pandemic-related fear is to validate the person's emotional experience, within reason. It is also important to help the person understand that fear can become one's ally rather than something to get rid of. When channelled appropriately, fear protects the individual as well as others by leading to protective behaviors such as hand washing, mask wearing, social distancing, and minimizing non-essential activities. Individuals with obsessive-compulsive disorder (OCD), many of whom hold contamination fears independent from COVID, may be particularly impacted by widespread concern over the coronavirus and may require specific adjustments to treatments (Fontenelle and Miguel 2020).

However, that being said, what can be modified is one's level of fear. Many are at a panic level of fear, meanwhile, the overwhelming majority of people are in fact not overly vulnerable to COVID. For those individuals, the following should become their guiding principle: COVID-19 will pass, humankind will survive, almost everyone will still be alive in its aftermath. The scientific evidence clearly supports this statement.

\section{Increased Media Consumption}

To varying degrees media (television, newspapers, social media, websites) coverage of events such as the COVID crisis increases one's perception of risk (Ropiek 2010). There is an old saying in the media: If it bleeds, it leads. The idea is the more horrific the story (e.g., the more frightening), the more likely it will be the headline and receive a lot of time or space in the way the media source covers it. Thus, media outlets are biased towards presenting the most threatening scenarios (people dying on ventilators, overfilled morgues, stories of the sickest patients, etc.) and not focused on alternative information which may present a more balanced or nuanced view (e.g., interviewing tens of thousands of people who had no or mild symptoms-overwhelmingly the typical course of COVID—or even people that were fairly sick who recovered). Consequently, these messages shape the general public's reality of the COVID crisis as overall more threatening than it is because of the biased information. It is also worth noting that it is not uncommon for false information to be spread by social media.

The news media can be viewed as a stimulus that triggers a certain response (immediate fear, skewed beliefs). Since this stimulus is something that everyone does in fact have control over the recommended strategy is stimulus control (Borkovec et al. 1983; McGowan and Behar 2013): reduce or eliminate exposure to the stimulus to prevent the undesirable response. While one needs to be informed, watching or reading the news for approximately 30-60 min per day should be sufficient to keep up with the necessary information. Accurate, non sensationally-oriented, print media is recommended. The Johns Hopkins Coronavirus Resource Center (https://coronavirus.jhu.edu/) meets these criteria and thus, is a highly recommended source to avoid unrealistic risk perception. News from local and state health departments which present factual and locally-oriented information are other reputable sources through which individuals can stay informed. 


\section{Perception of Increased Risk}

The topic of media information provides an excellent segue to perception of risk (danger). As discussed above, the media impacts beliefs about coronavirus in that it increases the perception of danger by presenting somewhat skewed information.

With regard to the experience of fear, for the most part, the more dangerous one perceives something, the greater the amount of fear that is experienced. Thus, the therapeutic goal is to help an individual develop accurate risk perception so that his or her fear is in proportion to the threat. The primary strategy to use is cognitive reappraisal (Lazarus and Alfert 1964; Gross and John 2003) to identify, examine, and when appropriate reappraise the situation.

Clearly, coronavirus is a threat. But like most threats, it is not the same for everyone. It is essential to keep in mind that fortunately most everyone will survive coronavirus. The following are several examples of how media consumption distorts a person's risk perception because of the way information is presented:

\section{Black and White Thinking}

You see COVID in extremes: either you do not get it, or you end up on a ventilator. There is no middle ground or shades of grey.

\section{Selective Attention}

You only focus on the worst cases. If a celebrity announces they are COVID positive but only have mild or no symptoms, you forget about him or her. But if a celebrity is very ill, that is the example that sticks in your brain.

\section{Catastrophizing}

You only focus on the worst outcome and personalize it: you are going to end up hospitalized with COVID just because that is a possibility. You lose the distinction between possibility (anything is possible) and probability (what is likely to happen based upon knowledge of base rates).

\section{Emotional Reasoning}

Because you feel anxious about this highly contagious virus that everyone else is anxious about, you assume it is dangerous- just because you feel it is. Watching media that displays cases and deaths like a scoreboard continues to exaggerate your anxiety further even though proportionally these are relatively low numbers compared to the entire population.

\section{Existential Concerns}

Central to human behavior is the motivation to stay aliveeven before the COVID crisis. If evolution designed the mind for anything it is to guide human survival. As mentioned above, fear is the invariable reaction that occurs when a threat is perceived and it guides our behavior towards maximizing survival (e.g., avoiding and escaping from danger, reducing the threat).

Some individuals seem to want certainty about their risk and likelihood that nothing bad will happen. Unfortunately, the fact is, there is no certainty that one will live beyond this moment. The probability for most everyone is that they will be here tomorrow-that is almost $100 \%$ certain. But since it is possible that something can happen at any moment, it is not a full $100 \%$. That is a point that must be accepted. Otherwise, attempting to gain certainty when it does not exist will exacerbate fear.

The following three points are important to keep in mind as we try to help individuals to accept the existential reality of our existence and consequently decrease fear driven by the attempt of obtaining certainty: (1) One can never be $100 \%$ certain that nothing will happen at any point in the future starting with now, (2) We take risks every day living our lives, and there is no alternative; very few of us would want to live in a bubble to maximize our safety and give up our lifestyle, (3) The best one can do is manage risks, not eliminate them fully.

In the COVID environment, one must ultimately confront death to move forward with some level of comfort. It is important to work towards accepting that we risk our survival every day so that we can live the life we want to live. The best one can do to manage their fear in the coronavirus landscape is to: (1) acknowledge this reality, (2) control whatever is reasonably possible (e.g., wear a mask while shopping, wash hands regularly, socially distance), and (3) move forward and live life. We attempt to get people not to deny the possibility of death, but to do their best to attend to managing risk and focus on realistic probabilities.

\section{Sadness and Depression}

Sadness (depression) is an emotional response that is primarily triggered by the occurrence of personal losses that are important to an individual and cannot easily be restored (e.g., loss of a job, failing a test) or in some instances are not able to be restored (irrevocable losses). These can be tangible losses, such as the death of a loved one or the breakup of a relationship, and they can be more abstract losses, such 
as failing to live up to one's expectations, or failing to have the marriage that one expected. The most severe form of sadness occurs when a person experiences, or perceives an experience, where the loss cannot be undone. The classic example is the death of a loved one (i.e., bereavement) where the loss is clearly irrevocable. However, it can also apply to a situation where a person believes (perceives) the loss is irrevocable; for example, a person who has a breakup of a relationship and truly believes he/she will never find anyone else.

With regard to the pandemic, what most are feeling are the effects of transient losses. For example, if someone misses seeing their friends during this period of social distancing and quarantine, the fact that the sadness is experienced is in fact because what was lost is important. The degree of sadness mirrors the importance of the loss.

When irrevocable loss occurs, or is perceived or believed to have occurred, the individual may go into a state of "resignation"- -an evolved response to preserve energy when using effort would be futile (the loss is irrevocable so the person needs to adjust rather than continue to attempt to restore it). However, when the resignation is triggered by perceptions of irrevocable losses (e.g., the world is never going to survive this pandemic, there is no way to fix the economy-it will be bad forever, I'll never see my friends again), it can lead to a state of resignation characterized by hopelessness and helplessness. These two states, as well as several other triggers of sadness, end up contributing to and maintaining the intense sadness, and people end up in a downward spiral from which it is difficult to escape. When helping people manage their sadness, the goal is to build their resilience, their ability to increase behavior to access what is important to them.

\section{Hopelessness}

Individuals may begin to experience an overwhelming lack of hope for the future as a result of the negative impact of the pandemic and the constant flooding of bleak news reports. While there are many unknowns, it is relatively certain that the transition back to our accustomed lives will not happen in the immediate future. This realization understandably adds to an individual's experience of hopelessness and depressive resigning behavior. As mentioned previously, stimulus control is recommended in order to combat feelings of hopelessness. Although it is unhelpful, attending to negative news is in our nature. Checking the news frequently may be contributing to hopelessness, even in hopes of more positive announcements. We are often reinforced to check more often due to intermittent segments of uplifting news, or anticipation of positive news, even if the overall message conveyed is overly negative. As aforementioned, individuals are recommended to limit COVID-19 related news consumption to a specified amount (i.e., once a day for $30 \mathrm{~min}$ ) and are urged to use accurate, non-sensationalized sources (Borkovec et al. 1983; McGowan and Behar 2013). Reappraisal of hopeless attitudes towards our current situation is also recommended as it is important to keep the reality in mind that for the most part these pandemic-related stressors are not permanent, although they may feel this way when one is in the midst of them. Focusing on the notion that humans are resilient and have previously overcome countless tragic crises (e.g., recessions, war, epidemics) is also imperative to provide a context of hope and survival-rather than resignation. (Note. For those in crisis, the National Suicide Prevention Lifelife (1-800-273-8255 or https://suicidepre ventionlifeline.org/chat/) is available 24/7.)

\section{Helplessness}

Relatedly, helplessness is both the belief that a situation will not change and the belief that one is unable to enact change on a situation. In other words, individuals who feel helpless believe that there is nothing they can do to change their undesirable circumstances; they have been convinced that their hopeless feelings are true. Unfortunately, helplessness may inadvertently create a self-fulfilling prophecy, that is one may behave in accordance with the predicted or expected outcome, thus creating a reality that confirms the faulty belief (e.g., if you do not study for an exam because you believe you will fail, you will in fact fail because you did not study, not because you were destined to fail). Additionally, feeling helpless may result in all-or-nothing thinking (e.g., "If I cannot fix the economy, I shouldn't bother ordering takeout from a local restaurant to support them"). Taken together, individuals are encouraged to reappraise allor-nothing thoughts (e.g., "The economy is doing poorly but I can do something to make a difference") and expand one's thinking to the "bigger picture" (e.g., "The economy is doing poorly but if everyone did something small, the scale of the impact would be enormous"). The pandemic might also be viewed as an opportunity to develop a new skill (e.g., sewing) that could be used to prompt supportive actions (e.g., distributing masks to others). With these changes in perspective, coupled with engaging in behaviors that are cognitively consistent, individuals can increase their sense of empowerment (i.e., the opposite of helplessness) and move out of the helpless frame.

\section{Non-bereavement Related Loss}

While many will experience grief related to a loss of a loved one due to COVID-19, this crisis has also introduced an array of non-bereavement related loss (e.g., loss of social connection, jobs, normalcy, economic stability). These losses have been found to instill a similar grief reaction, especially when the loss is directly associated with one's 
identity (Papa et al. 2014). The level and intensity of grief varies per individual, as do the order of the stages of grief (i.e., denial or avoidance, anger, bargaining, sadness, and acceptance). While grief reactions are expected as a result of pandemic-related loss, research on grief has indicated several strategies to cope with and process these losses. Expressive writing in the form of identifying and naming the experienced loss can help individuals increase awareness of the associated emotions and recognize previous strategies that were effective in coping with that emotion in the past. After naming the losses, outlining ways to move forward in or amend those loss areas can help individuals accept their loss and work towards reproducing what has been lost (e.g., after job loss, reading or attending webinars on new innovations of your field; Briggs and Pehrsson 2008). Additionally, social support is largely indicated in grief interventions. While social distancing limits physical social contact, grieving individuals can still access social support. Phone and online platforms can increase the social support experience through voice or video methods, in comparison to texting which can be less personal.

\section{Loneliness}

Global quarantine and social distancing regulations have resulted in significantly limited social contact. As a result, many individuals have started to socially and physically resign and are overwhelmed by upsetting thoughts related to loneliness. Although these reactions are understandable, they typically exacerbate feelings of social disconnection. Instead, individuals are encouraged to validate their feelings, challenge unhelpful thoughts, and problem solve in order to increase connection in novel ways (Sanderson et al. 2020). Helpful strategies that can be used to think about the current regulations in a more balanced manner include: relabeling the current guidelines from "social distancing" to "physical distancing;" remembering that individuals are apart now so they can be together later; and reframing the situation as a time to focus on, build, and/or create meaningful relationships. Several practical ways to increase connection include but are not limited to: Zoom video chats, facetime, Netflix movie parties, handwritten letters, video games, virtual exercise classes, online forums, online support groups, virtual game nights, virtual paint nights, virtual book clubs, hugging a stuffed animal, and purchasing long distance friendship lamps.

\section{Loss of Reinforcement}

Loss of income and loss of social reinforcement are two ways the pandemic has decreased reinforcement among individuals (Pfefferbaum and North 2020). Such losses can decrease effective behaviors leading to psychological distress and increase ineffective behaviors which pose health risks (e.g., socially isolating, avoidance, washing hands irregularly; Brooks et al. 2020). Engaging in a range of activities that balance pleasure, mastery, and social connection is often most helpful for boosting reinforcement and promoting positive moods. For example, cooking different meals at home can be inherently enjoyable (i.e., flavorful food), result in a sense of achievement (i.e., learning a new recipe), and promote social connection (i.e., sharing the recipe or meals with others). With regards to health behaviors that individuals may struggle to implement (e.g., wearing a mask, handwashing), basic principles of contingency management can be used to increase the desired behavior. Individuals can set goals for themselves, track their progress, and reward themselves when goals are met. This can be helpful to increase behaviors that are not immediately pleasurable, but are important to do in the current context.

\section{Loss of Meaning}

As the pandemic continues, many perceive a sense of meaninglessness due to uncontrollable circumstances (e.g., unemployment, decreased social interaction, and various other role losses). The disruptions in daily life lead many individuals to feel a loss of purpose which they normally derive from involvement in careers, caregiving, or other activities. Therapeutic approaches that prioritize meaning have been shown to be effective in improving quality of life (Breitbart et al. 2018). Relatedly, as aforementioned, CBT emphasizes increasing engagement in pleasant and masteryoriented activities as a general mood management strategy. A helpful strategy is for individuals to mourn the unavoidable role-related losses while simultaneously shifting focus to aspects in their control. Individuals can connect purposefully with the world by engaging in hobbies, learning new skills, keeping a pandemic journal, or helping others. Small contributions like phoning someone who lives alone, shopping for others, or donating to causes more directly (e.g., blood drives, buying supplies, fundraising) hold tremendous potential for cultivating a sense of meaning and are activities that can be safely conducted during social distancing efforts.

\section{Rumination}

Individuals are being exposed to a deluge of negative information about a problem that—at least in the short term-has no clear solution. Additionally, given that this negative information is relevant to the current public health crisis, it is unsurprising that individuals are more likely than ever to attend to it. Initially, increased attention to negative information may appear to be solution-focused or goal-directed thinking and, due to the current crisis, individuals may be more likely to continue engaging in such behavior. However, individuals can 
easily become stuck in a cycle of ruminative (i.e., brooding and dwelling) thoughts about the current situation which results in exacerbated emotional distress, agitation, and/or sadness. While this reaction is unsurprising, self-monitoring is recommended to help individuals identify specific external cues (e.g., media consumption, speaking with a friend or relative who catastrophizes), emotional states (e.g., loneliness, sadness, boredom), and locations or times of day (e.g., isolated in home office, late at night) when ruminative thinking is more likely to occur again. Increased awareness of cognitive, behavioral, and environmental triggers then creates a personalized guide for what cues the individual should remove or avoid from their environment (e.g., limiting news intake, schedule "socializing" breaks throughout the workday). However, it is important to note that if the "socializing break" is a virtual one; changing one's physical location during the call is crucial to differentiating this break from the workday (i.e., the work environment in one's home).

\section{Depressive Thinking}

Thoughts of hopelessness and helplessness, rumination and worry, and overall negative thinking are expected when individuals are exposed to an overwhelming amount of negative information regarding the pandemic (i.e., death statistics, case increases, economic collapse). In combination with a predisposition to negativity bias, (Ito et al. 1998) a pandemic undoubtedly exacerbates depressive thinking (i.e., negatively oriented news focused on dangers and losses). Practicing gratitude is a recommended strategy that may help lessen our negativity bias by transferring a portion of our attention to positive stimuli. Individuals can begin to assess what they are thankful for, and deliberately attend to simple moments of pleasure each day to transfer focus to contentment rather than loss. Being mindful during pleasurable activities (i.e., recognizing emotions) and when engaging one's senses (i.e., taste of a meal) can help increase awareness of moments for which one can be grateful. Similarly, increasing awareness of negative thoughts and of their frequency can help individuals notice the onset of the thought, and engage in a positively salient activity in order to halt the depressive thinking cycle. Thought monitoring and cognitive reappraisal are particularly powerful ways to combat depressive thinking as outlined above. Finally, engaging in physical exercise (see below for section on sedentary behavior) can be an excellent way to change thoughts through behaviors.

\section{Other Negative Psychological Experiences}

\section{Guilt}

Guilt has been a frequent consequence of the pandemic. Some may discover they tested positive for COVID after they were in contact with others, some may be struggling with not being able to actively help during this crisis, healthcare workers may not be able to save patients, and some may be feeling more privileged than others. As a result, individuals are blaming themselves, questioning their behaviors, punishing themselves (whether intentionally or not), and resigning. To manage feelings of guilt, individuals are often encouraged to identify the source of their guilt, evaluate how much responsibility they have for it, take the appropriate amount of responsibility, make reparations for any harm they might have caused, and ultimately forgive themselves. Additionally, engaging in prosocial behaviors is recommended (e.g., sending a care package to someone struggling, ordering takeout from local restaurants, reaching out to someone who might be lonely, making a donation) to help with thoughts about not doing enough and increasing one's sense of empowerment.

\section{Shame}

Shame occurs when individuals engage in reputation-damaging behaviors or those that have a heavy cost to society (Cibich et al. 2016). In the current context, a positive COVID status can be shame-inducing due to the stigma associated with being contagious, as well as the costs it has to society (i.e., potential of infecting others). Shame can be problematic if the feeling is so intense that it negatively affects self-esteem and mental health (e.g., feeling worthless despite taking adequate quarantine precautions). Shame can also lead to avoidance or ineffective behaviors (e.g., not telling housemates about positive test results). The first step to manage shame is to determine if it is justified or not. Shame experienced from disease status per se is not justified, but other behaviors (e.g., not wearing a face covering when symptomatic) can be. Shame can be functional if it guides one to rectify a negative behavior (i.e., informing others, taking precautions). For unjustified or excessive shame, identifying and acknowledging the emotion is the first step to reduce the intensity. Next, individuals can identify behaviors driven by the emotion and engage in the opposite behavior (the 'Opposite Action' skill in Dialectical Behavior Therapy; Rizvi and Linehan 2005). To reduce the effects that shame has on self-esteem, it is important to first differentiate qualities of oneself that do not change from having the virus, and to identify and reframe "shame thoughts." For example, instead of not disclosing testing positive for the virus because of thoughts that they are "bad" or "dirty", individuals can be encouraged to disclose it to appropriate people in their lives and see themselves as "brave" and "proud" for socially isolating and taking steps to protect others, which is valuable to society. 


\section{Unrealistic Personal Expectations}

As aforementioned, the media frequently highlights biased information and often focuses on those who appear to be maximizing productivity and accumulating accolades during this pandemic. The tendency to make comparisons between these featured individuals and ourselves can lead to personal feelings of inadequacy, as well as attempts at radical behavioral changes with goals that may be difficult to attain, resulting in increased feelings of failure and reduced selfesteem (Vogel et al. 2014). While it is common to engage in these comparisons, it may be more helpful to practice reappraisal, recognizing that those featured likely represent only a small percentage of the population, and that what is shown is highly selected for, thus leading to a biased perception (i.e., sitting on the couch and watching television is an activity many individuals participate in, but is not broadcasted as it is not especially attention-grabbing). Additionally, rather than setting broad, ambitious goals that may be exceedingly difficult to achieve, focus should be on setting goals that are smaller, observable, specific, and measurable. Research has shown that two key factors in maintaining commitment in working towards a goal include (1) the belief that one can realistically attain the goal, and (2) importance given to the outcome expected as a result of attaining the goal (Locke and Latham 2002). Thus, it is critical to set goals that are challenging enough to feel a sense of accomplishment when achieved, while not being overly onerous. Lastly, specificity aids in accountability, and detailing what the goal is and how to measure it, as well as scheduling the time and day during the week to engage in goal-focused activity, can help facilitate action towards reaching a goal.

\section{Frustration}

Collectively, the population is encountering an increase in daily and repeated frustration (i.e., achieving one's goals is either more difficult or currently impossible). Frustrations may be the result of external (e.g., experiencing interrupted internet connection), internal (e.g., low self-confidence after failing to meet work deadlines), or particularly salient (e.g., specific cleaning supplies consistently being out of stock) daily experiences. Experiencing frustration may lead to an increase in negative mood and result in maladaptive thinking such as, catastrophizing (e.g., "I am the worst employee ever"), overgeneralizing (e.g., "My internet never works"), and fortune telling (e.g., "If the cleaning product was out of stock at the first store, none of them will have what I need"). Additionally, an individual's interpretation of any given event is influenced by a number of factors (e.g., current emotions, life circumstances, relationships, the weather, etc.) and tends to change over time. Given the heightened level of emotional distress an individual may be experiencing, interpretation of neutral or even somewhat negative events as catastrophic is expected. Cognitive reappraisal is recommended to shift the individual's perception of the increased meaning and significance of these negative events and ultimately decrease negative emotion. Furthermore, encouraging individuals to reframe the situation which led to frustration in a more positive way or as an opportunity to increase effortful action and goal-directed behavior (e.g., problem solving) will be helpful.

\section{Claustrophobia}

We propose the existence of a condition that can best be described as pandemic-induced claustrophobia; not claustrophobia in the traditional sense of the psychological disorder, but a unique feeling of discomfort related to being "trapped" at home. This "condition" may be particularly prevalent for those living in apartments, in cities where ample space is rare, and/or with several other people. Such individuals may feel stir-crazy yet exhausted, exhibit negative affect, and be tempted to break social distancing policies. An effective mental strategy to combat pandemic-specific claustrophobia is cognitive reappraisal. In the current situation, this tactic can take the form of changing one's attributions for the quarantine: viewing staying inside as an intrinsically motivated behavior as opposed to a punitive decree from an external source. An example strategy is thinking of a specific person one knows who is a member of a high-risk population, and telling oneself that one's decision to stay inside is in the service of protecting this person. Alternatively, some individuals may interpret stay-at-home orders as prohibitive of going outside even for fresh air or exercise. This type of appraisal may contribute to a feeling of being "trapped." By consciously reframing the situation (e.g., "I should limit my contact with others, but going for walks outside is acceptable"), individuals can feel a greater sense of agency and control over their current circumstances and reduce claustrophobic feelings that may be exaggerated from these misinterpretations.

\section{Chronic Stress}

Feeling stressed or overwhelmed is one of the most commonly reported emotional experiences of the pandemic; a cumulative effect of being faced with a variety of new challenging circumstances. This response is unsurprising, but also problematic, given that the body responds to stressful external circumstances with increased production of the hormone cortisol. Elevated levels of cortisol are associated with numerous negative health consequences - including, ironically, diminished immune response. Stress management is therefore crucial for both mental and physical well-being during the pandemic, and one major tool for combating the negative effects of stress is 
meditation (see section below on Lifestyle Factors for additional tools to help manage stress, most notably the section on exercise). In fact, numerous studies over recent decades have demonstrated the benefits of meditation for many facets of physical, emotional, and mental health (Lynch et al. 2018). This practice may be particularly relevant at this time given that many individuals do not have access to their regular stress relief outlets (going to the gym, gathering with friends, etc.). An abundance of meditation-related offerings exist online, from formal training programs to guided mindfulness exercises. Thus, the first step in establishing a meditation practice is taking the time to explore different meditation resources and determine what type of meditation one finds to be the best personal "fit." By cultivating an enjoyable and sustainable practice, individuals are more likely to remain consistent in their meditation, which is key for reaping maximum health benefits.

\section{Irritability and Conflict}

More than ever, individuals are working from home and spending more time at home, leading to increased contact with families and roommates. Stress-induced negative emotions, boredom, and irritability naturally contribute to more conflict in households. Of note, incidents of domestic violence have substantially increased worldwide since the onset of stay-athome orders (Boserup et al. 2020). Self-care methods and relaxation strategies can reduce irritability in the long-term, potentially making conflicts less likely to occur. Conflict may arise from reductions in privacy or from disagreements about safety precautions due to variability in risk tolerance. Individuals are encouraged to recognize that others differ in the extent to which they desire solitude as well as their comfort level in taking health precautions. While there are certain public health guidelines for navigating health behaviors, ideally households can be respectful in conversations and seek a compromise. Specific communication strategies are also useful for anticipating, reducing, and navigating interpersonal conflicts. For example, it can be helpful to first become aware of emotions in the moment and take a "time out" as necessary (e.g., counting to 10 , leaving the room) before responding. If tensions are still high, deciding on a future time to have discussions can be effective. Communicating using "I" statements and making requests instead of demands are other helpful tactics for more effectively resolving disputes. (Note. For anyone affected by abuse and needing support, call 1-800-799-7233, go to thehotline.org, or text LOVEIS to 1-866-331-9474).

\section{Lifestyle Factors}

\section{Lack of Sunlight}

Research suggests a strong positive relationship between sunlight and serotonin levels (Lambert et al. 2002), a neurotransmitter central to biological explanations of depression. The pandemic cancelled many outdoor events and shuttered businesses, workplaces, and other establishments. As a result, many are spending more time indoors, away from sunlight. Sunlight can promote positive moods, so individuals should aim to get at least $30 \mathrm{~min}$ of sunlight each day. This practice could take the form of walking around neighborhoods, sitting outside, or engaging with nature (e.g., hiking, walking near water). Several of these suggestions can be combined with other pleasurable activities (e.g., reading, listening to podcasts, making telephone calls). If outdoor activity is still not possible, light therapy lamps can also have antidepressant benefits (Kripke 1998).

\section{Poor Sleep}

Social distancing efforts are disrupting routines, leading to less daily structure and negatively impacting sleep. Many individuals also report increased screen time and anxious thoughts at bedtime, making insomnia a problematic consequence of the pandemic. Decades of research show that adequate sleep is necessary for physical health, immune functioning, and mental well-being. CBT for insomnia involves working with dysfunctional beliefs about sleep, utilizing stimulus control, and practicing good sleep hygiene. In addition to getting sunlight and physical exercise throughout the day, keeping a regular sleep schedule, engaging in a relaxing wind down routine, and limiting electronic use before bed are all helpful strategies. If individuals cannot sleep at night, they should go to another part of their home and do a calming activity until they feel sleepy, ensuring that bedrooms are used for sleep only. Finally, anxious thoughts should be dealt with prior to bed time (e.g., worry time) and dysfunctional beliefs about sleep (e.g., catastrophic outcomes resulting from not achieving a "perfect" night's sleep) can be addressed with cognitive reappraisal and psychoeducation.

\section{Sedentary Behavior}

Telework and home entertainment-common during the pandemic - lead to prolonged sitting and a normalization of sedentary lifestyles. Social distancing may greatly reduce individuals' activity levels, since gyms are closed and organized team sports are cancelled. Even before the pandemic, many people failed to get the $150 \mathrm{~min} /$ week of aerobic 
exercise recommended by the American Heart Association. However, physical activity promotes positive moods and can be protective against depression (Schuch et al. 2018) so becoming more active is very important. Activities such as walking, running, hiking, or indoor activities (e.g., fitnesscentered video games, Zoom yoga classes) are all helpful. Individuals who are beginning new exercise routines should consider physical activity as they would any new habit, emphasizing starting small and being consistent.

\section{Changes in Eating Habits}

For many, the pandemic has caused a prolonged increase in stress, which often leads to an increase in the consumption of highly palatable high-fat and high-sugar foods (Adam and Epel 2007). The intake of calorically-dense foods has been found to stimulate the reward center of the brain, thus reinforcing this behavior by temporarily reducing negativeaffect. However, consequences of eating such foods include inflammation in the body, which has been linked to various health conditions such as depression (Kiecolt-Glaser 2010). Therefore, the strategy of eating such foods to manage stress and increase feelings of well-being is counterproductive. Instead, more prudent strategies involve cognitive appraisal, deliberate behavioral changes, and awareness around stimulus control. For example, avoid purchasing these highly caloric foods, since reduced access will likely equate to decreased consumption. Additionally, taking advantage of time at home by planning and preparing meals in advance can help override the tendency to reach for highly caloric foods when hungry or stressed.

\section{Changes in Technology Use}

While social connection is of utmost importance during a period of social distancing, a distinct set of challenges related to boundary infringement emerged within the social lives of those asked to stay at home, work at home, and socialize from home. This change is in part a result of a prevalent aspect of the "new normal" which includes the utilization of videoconferencing platforms (e.g., Zoom) for work meetings, academic classes, and social calls.

\section{Boundary Infringement}

Individuals working from home were asked to do so within the context of unclear expectations (e.g., increased availability) and increased demands (e.g., homeschooling children). Unsurprisingly, individuals struggling with the effects of boundary infringement may react by either withdrawing and avoiding particularly aversive work-related tasks or by being overly accommodating and working increased hours. However, neither of these responses is sustainable; avoidance often leads to increased anxiety and overworking often leads to burnout. A helpful strategy is to develop and implement a behavior management plan in order to set a metaphorical boundary between work and home, given that the physical boundary is no longer in place.

\section{Zoom Fatigue}

In addition, despite the usefulness of video conferencing software, a newly-recognized phenomenon is "Zoom fatigue," described in the media as a feeling of being disengaged during video conferences and/or mentally drained after signing off. Virtual meetings lack many of the nuances that make in-person interactions feel connected and organic, while also presenting challenges such as internet connectivity issues, background noises, and awkward pauses or moments of cross-talk. As a result, those with many Zoom obligations may emotionally withdraw, becoming less participative in work meetings and choosing not to join video calls with friends despite already feeling socially isolated. This unique brand of burnout can be combated with specific behavioral strategies aimed to help individuals re-engage with their Zoom activities by treating virtual get-togethers the same way they would treat those events in real life. For example, to get into a "work" mindset, individuals should keep the same pre-work morning routine as they held before the pandemic, create one designated workspace in their home, and practice staying engaged in meetings by asking questions. To help maintain enthusiasm about virtual social activities, individuals should seek to create a clear delineation between work-related video calls and social video calls. This distinction can be accomplished by using different video apps for each kind of call, changing out of one's work clothes at the end of the day and into clothes one would normally wear for social events, and not taking social calls in one's designated workspace area. Finally, individuals may benefit from reaching out to family and friends to process feelings of burnout, and recruiting loved ones to help hold them accountable.

\section{Social Connectedness}

On the other hand, in the service of "staying connected," individuals may feel pressured to respond quickly to alerts on mobile devices or participate in virtual get togethers even when their time might be better spent in solitude. Others may participate in virtual social events only to find themselves begrudgingly talking about news, politics, or the pandemic. For those who feel sufficiently connected, communicating clearly about feelings and intentions using assertive statements is important. Setting appropriate boundaries with electronic communications can also be very helpful, either by silencing mobile notifications, lengthening delays before 
responding to others, or skipping virtual meetups all together if feeling socially "worn out." Whatever approach is taken, increasing agency within communications may decrease feelings of resentment and, as a result, make socialization more fulfilling.

\section{A Preliminary Intervention}

To address these various concerns, our clinic has created a two-session consultation service for psychological distress related to COVID (for materials, see Limowski et al. 2020). The consultation service was advertised on the university clinic's website so that the link could be easily shared by students and faculty. The description is as follows: "In order to offer our expertise in helping individuals cope with psychological distress and emotional difficulties related to COVID-19, the staff of the Anxiety and Depression Clinic (Director: William C. Sanderson, $\mathrm{PhD}-\mathrm{a} \mathrm{NY}$ State Licensed Psychologist) is offering a consultation series consisting of two 60-min online sessions with an ADC staff member." Clients are asked to provide responses to questions that assess their current and pre-pandemic levels of fear, sadness, and emotional distress on a 10-point scale, identify and provide information about their most challenging pandemicrelated circumstance, and provide some information about their mental health functioning (e.g., past/current medication use, therapy, diagnosis). Sessions are scheduled by email and take place over HIPAA compliant Zoom.

Our first session is based on the single-session model used by Schleider (2020) and involves targeting one or two main problem areas with cognitive behavioral strategies. Our clinic additionally offers a follow-up session one week later to further assist clients in utilizing strategies previously discussed. At time of writing, four clients have completed our brief consultation service. Issues addressed most commonly involved managing job-related stress, setting healthy boundaries, practicing sleep hygiene, managing excessive worry related to uncertainties, and reducing alcohol consumption. While of course, these data are very preliminary given the small sample size, it is worth noting that self-report assessment conducted following the second session indicates that the consultation service was of value clients. Specifically, clients reported experiencing approximately $33 \%$ reductions in fear, sadness, and general distress over the course of one week following the implementation of the recommended strategies. All clients rated the intervention as "very helpful" in addressing their concerns and developing an action plan (both rated as 5 on a 5 point scale). Similarly, therapists conducting these sessions believed they were effective in administering a very focused, useful intervention. These data are promising with regard to delivering a remote, scalable, and effective intervention for those suffering from pandemicrelated psychological distress.

\section{Resilience and Posttraumatic Growth}

As is evident, many individuals are struggling with a plethora of COVID-related triggers; however, a variety of therapeutic strategies (indicated above) can be helpful in managing the resulting distress. In fact, highly stressful life events that are often associated with periods of grief and loss are also typically associated with hopeful periods of readjustment and healing (Tedeschi and Calhoun 2004). Thus, during the COVID crisis, it is possible that many individuals will convey healthy response patterns-some may exhibit resilience (i.e., maintaining baseline functioning in the context of disruption) and others may even experience posttraumatic growth (i.e., improving baseline functioning in the context of disruption) as a result of dealing with a new reality. Generally, individuals report experiencing at least one positive change after a potentially traumatic event (e.g., an increased appreciation for life, stronger and closer relationships; Tedeschi and Calhoun 2004). Therefore, dealing with the abrupt and ongoing changes of the pandemic can actually have positive psychological effects over time.

During the COVID crisis, it is understandable that individuals feel down and distressed at times; however, expecting to only feel this way won't allow for the possibility of even momentary health and wellness. Instead, a curious, open, and appreciative mindset can promote self-maintenance and growth. Commuting time can be repurposed in new and valuable ways, individuals can focus on meaningful activities they may not have had time for previously, and new hobbies can be embraced and potentially result in longterm lifestyle changes. As regulations are slowly lifted, individuals can also begin to practice appreciating life's day to day activities that may have been previously interpreted as mundane or even aversive (e.g., grocery shopping, sitting in traffic, running errands). Additionally, being proactive and deliberate with one's actions towards growth (e.g., setting goals) and embracing resilience resources (e.g., individual characteristics and skills, social support within communities, finding a sense of meaning/purpose) are also particularly helpful in cultivating progress (Rosenberg 2020). Importantly, while some may experience resilience and growth, expecting this outcome at all times is unreasonable and unhelpful. There is no "right" way to cope with the pandemic, and growth isn't essential to survive. Instead, individuals need to simply do what works best for them during these challenging times.

In sum, although the COVID crisis has resulted in numerous problems, resilience and growth are not only possible, but probable. However, resilience requires a "growth 
mindset"- one which acknowledges the negative but also looks for opportunities for improvement.

\section{Conclusion}

The COVID-19 crisis is expected to have an enormous negative impact upon the mental health of the world's population (Marques et al. 2020; Strakowski et al. 2020). Unfortunately, the mental health system in the U.S. - and perhaps other places in the world - is not well poised to deal with the psychological distress associated with the pandemic. Given the novelty of this situation, specific treatments have not yet been developed to target the pandemic-related triggers that are resulting in a significant amount of stress. In addition, the present system may not be able to meet the current and future increased need for mental health services and thus, scalable interventions will be necessary to better distribute the resources available to a greater number of individuals.

It is important to note that based upon responses to similar stressors, most individuals, even if acutely distressed, are likely to recover on their own once the pandemic passes (Rauch et al. 2020). Indeed, humans are resilient! Nevertheless, providing evidence-based cognitive behavioral emotion regulation skills to those experiencing significant distress in the moment has obvious value in that it can facilitate increased comfort as well as decrease the likelihood of more severe problems emerging down the road. If mental health professionals view all psychological distress as a "normal" response to the pandemic, and thus not requiring intervention, this may ultimately lead to significantly worse mental health outcomes for many individuals down the road. As a result, identification and an appropriate level of treatment for those with pandemic-related mental health issues now-ranging from providing self-help information to brief specific interventions to longer term psychotherapeutic treatment-is critical to prevent the development of a mental health pandemic that lasts beyond the COVID-19 crisis.

Data Availability The data were not collected.

\section{Compliance with Ethical Standards}

Conflict of interest The authors declared that they have no conflict of interest.

Informed consent There was no need for informed consent.

\section{References}

Adam, T. C., \& Epel, E. S. (2007). Stress, eating and the reward system. Physiology \& Behavior, 91(4), 449-458. https://doi.org/10.1016/j. physbeh.2007.04.011.
Borkovec, T. D., Wilkinson, L., Folensbee, R., \& Lerman, C. (1983). Stimulus control applications to the treatment of worry. Behaviour Research and Therapy, 21(3), 247-251.

Boserup, B., McKenney, M., \& Elkbuli, A. (2020). Alarming trends in US domestic violence during the COVID-19 pandemic. The American Journal of Emergency Medicine. https://doi.org/10.1016/j. ajem.2020.04.077.

Breitbart, W., Pessin, H., Rosenfeld, B., Applebaum, A. J., Lichtenthal, W. G., Li, Y., ... Fenn, N. (2018). Individual meaning-centered psychotherapy for the treatment of psychological and existential distress: A randomized controlled trial in patients with advanced cancer. Cancer, 124(15), 3231-3239. https://doi.org/10.1002/ cncr.31539.

Briggs, C. A., \& Pehrsson, D. E. (2008). Use of bibliotherapy in the treatment of grief and loss: A guide to current counseling practices. Adultspan Journal, 7(1), 32-42. https://doi. org/10.1002/j.2161-0029.2008.tb00041.x.

Brooks, S. K., Webster, R. K., Smith, L. E., Woodland, L., Wessely, S., Greenberg, N., et al. (2020). The psychological impact of quarantine and how to reduce it: Rapid review of the evidence. The Lancet, 395(10227), 912-920. https://doi.org/10.1016/S0140 -6736(20)30460-8.

Centers for Disease Control and Prevention. (2020, June 10). Mental health household pulse survey. https://www.cdc.gov/nchs/covid 19/pulse/mental-health.htm.

Cibich, M., Woodyatt, L., \& Wenzel, M. (2016). Moving beyond "shame is bad": How a functional emotion can become problematic. Social and Personality Psychology Compass, 10(9), 471-483. https://doi.org/10.1111/spc3.12263.

Da, B. L., Im, G. Y., \& Schiano, T. D. (2020). COVID-19 hangover: A rising tide of alcohol use disorder and alcohol-related liver disease. Hepatology. https://doi.org/10.1002/hep.31307.

Dusharme, J. (2020, June 11). How to soothe your 're-entry anxiety' as COVID-19 lockdowns lift. Time. https://time.com/5850143/covid -19-re-entry-anxiety/.

Fiorillo, A., \& Gorwood, P. (2020). The consequences of the COVID19 pandemic on mental health and implications for clinical practice. European Psychiatry, 63(1), e32, 1-2. https://doi. org/10.1192/j.eurpsy.2020.35.

Fontenelle, L. F., \& Miguel, E. C. (2020). The impact of coronavirus (COVID-19) in the diagnosis and treatment of obsessive-compulsive disorder. Depression \& Anxiety, 37, 510-511. https://doi. org/10.1002/da.23037.

Gross, J. J., \& John, O. P. (2003). Individual differences in two emotion regulation processes: Implications for affect, relationships, and well-being. Journal of Personality and Social Psychology, 85(2), 348-362. https://doi.org/10.1037/0022-3514.85.2.348.

Ito, T. A., Larsen, J. T., Smith, N. K., \& Cacioppo, J. T. (1998). Negative information weighs more heavily on the brain: The negativity bias in evaluative categorizations. Journal of personality and social psychology, 75(4), 887. https://doi. org/10.1037//0022-3514.75.4.887.

Kiecolt-Glaser, J. K. (2010). Stress, food, and inflammation: Psychoneuroimmunology and nutrition at the cutting edge. Psychosomatic Medicine, 72(4), 365-369. https://doi.org/10.1097/ PSY.0b013e3181dbf489.

Kripke, D. F. (1998). Light treatment for nonseasonal depression: Speed, efficacy, and combined treatment. Journal of Affective Disorders, 49(2), 109-117. https://doi.org/10.1016/S0165 -0327(98)00005-6.

Lambert, G. W., Reid, C., Kaye, D. M., Jennings, G. L., \& Esler, M. D. (2002). Effect of sunlight and season on serotonin turnover in the brain. The Lancet, 360(9348), 1840-1842. https://doi.org/10.1016/ S0140-6736(02)11737-5.

Lazarus, R. S., \& Alfert, E. (1964). Short-circuiting of threat by experimentally altering cognitive appraisal. Journal of Abnormal and 
Social Psychology, 69(2), 195-205. https://doi.org/10.1037/h0044 635.

Limowski, A. R., Krychiw, J. K., Arunagiri, V., \& Sanderson, W. C. (2020). COVID-19 mental health consultation service: Intervention Protocol. PsyArXiv.https://osf.io/7caqg/.

Locke, E. A., \& Latham, G. P. (2002). Building a practically useful theory of goal setting and task motivation: A 35-year odyssey. American Psychologist, 57(9), 705-717. https://doi. org/10.1037/0003-066X.57.9.705.

Lynch, J., Prihodova, L., Dunne, P. J., McMahon, G., Carroll, A., Walsh, C., et al. (2018). Impact of mantra meditation on health and wellbeing: A systematic review protocol. European Journal of Integrative Medicine, 18, 30-33. https://doi.org/10.1016/j.eujim .2018.01.008.

Marques, L., Bartuska, A. D., Cohen, J. N., \& Youn, S. J. (2020). Three steps to flatten the mental health need curve amid the COVID-19 pandemic. Depression and Anxiety, 37(5), 405-406. https://doi. org/10.1002/da.23031.

McGowan, S. K., \& Behar, E. (2013). A preliminary investigation of stimulus control training for worry: Effects on anxiety and insomnia. Behavior Modification, 37(1), 90-112. https://doi. org/10.1177/0145445512455661.

Panchal, N., Kamal, R., Orgera, K., Cox, C., Garfield, R., Hamel, L., et al. (2020). The implications of COVID-19 for mental health and substance use. San Francisco: Henry J. Kaiser Family Foundation.

Papa, A., Lancaster, N. G., \& Kahler, J. (2014). Commonalities in grief responding across bereavement and non-bereavement losses. Journal of Affective Disorders, 161, 136-143. https://doi. org/10.1016/j.jad.2014.03.018.

Pfefferbaum, B., \& North, C. S. (2020). Mental Health and the Covid19 Pandemic. New England Journal of Medicine. https://doi. org/10.1056/NEJMp2008017.

Rajkumar, R. P. (2020). COVID-19 and mental health: A review of the existing literature. Asian Journal of Psychiatry, 52, 1-5. https:// doi.org/10.1016/j.ajp.2020.102066.

Rauch, S. A., Simon, N. M., \& Rothbaum, B. O. (2020). Rising tide: Responding to the mental health impact of the COVID-19 pandemic. Depression and Anxiety, 37, 505-509. https://doi. org/10.1002/da.23058.

Rizvi, S. L., \& Linehan, M. M. (2005). The treatment of maladaptive shame in borderline personality disorder: A pilot study of "opposite action". Cognitive and Behavioral Practice, 12(4), 437-447. https://doi.org/10.1016/S1077-7229(05)80071-9.

Ropiek, D. (2010). How Risky Is It, Really?: Why Our Fears Don't Always Match the Facts. United States: McGraw-Hill.

Rosenberg, A. R. (2020). Cultivating deliberate resilience during the Coronavirus Disease 2019 pandemic. JAMA Pediatrics. https:// doi.org/10.1001/jamapediatrics.2020.1436.
Sanderson, W. C., Arunagiri, V., Funk, A. P., Ginsburg, K. L., Klurfeld, Z. B., Krychiw, J. K., et al. (2020). Coping with fear and sadness during a pandemic. PsyArXiv. https://doi.org/10.17605 /OSF.IO/3DZ69.

Schuch, F. B., Vancampfort, D., Firth, J., Rosenbaum, S., Ward, P. B., Silva, E. S., ... Fleck, M. P. (2018). Physical activity and incident depression: A meta-analysis of prospective cohort studies. American Journal of Psychiatry, 175(7), 631-648. https://doi. org/10.1176/appi.ajp.2018.17111194.

Schleider, J. L. (2020). Single-session consultation for emotional and behavioral health. PsyArXiv. https://doi.org/10.17605/OSF.IO/ XNZ2T.

Schleider, J. L., Sung, J., Bianco, A., Gonzalez, A., Vivian, D., \& Mullarkey, M. C. (2020, May 29). Open pilot trial of a singlesession consultation service for clients on psychotherapy waitlists. Retrieved from https://psyarxiv.com/fdwqk.

Strakowski, S., Keller, A., \& Ghaemi, N. (2020, May 8). New projections on suicide, substance abuse, and COVD-19 [Video]. Medscape. https://www.medscape.com/viewarticle/929632.

Substance Abuse and Mental Health Services Administration. (2019). Key substance use and mental health indicators in the United States: Results from the 2018 National Survey on Drug Use and Health (HHS Publication No. PEP19-5068, NSDUH Series H-54). Rockville, MD: Center for Behavioral Health Statistics and Quality, Substance Abuse and Mental Health Services Administration. Retrieved from https://www.samhsa.gov/data/.

Sun, L. (2020, April 9). CDC director warns second wave of coronavirus is likely to be even more devastating. The Washington Post. https://www.washingtonpost.com/health/2020/04/21/coronaviru s-secondwave-cdcdirector/.

Tedeschi, R. G., \& Calhoun, L. G. (2004). Posttraumatic growth: conceptual foundations and empirical evidence. Psychological Inquiry, 15(1), 1-18. https://doi.org/10.1207/s15327965pli150 1_01.

Vogel, E. A., Rose, J. P., Roberts, L. R., \& Eckles, K. (2014). Social comparison, social media, and self-esteem. Psychology of Popular Media Culture, 3(4), 206-222. https://doi.org/10.1037/ppm00 00047.

Volkow, N. D. (2020). Collision of the COVID-19 and addiction epidemics. Annals of Internal Medicine. https://doi.org/10.7326/ M20-1212.

Publisher's Note Springer Nature remains neutral with regard to jurisdictional claims in published maps and institutional affiliations. 